\title{
Kidney morphological parameters measured using noncontrast- enhanced steady-state free precession MRI with spatially selective inversion recovery pulse correlate with eGFR in patients with advanced CKD
}

\author{
Tadashi Otsuka $^{1,2} \cdot$ Yoshikatsu Kaneko $^{1}$ (D) Yuya Sato $^{1} \cdot$ Ryohei Kaseda $^{1} \cdot$ \\ Ryuji Aoyagi $^{2} \cdot$ Suguru Yamamoto $^{1} \cdot$ Shin Goto $^{1} \cdot$ Ichiei Narita $^{1}$
}

Received: 31 January 2017/Accepted: 13 April 2017/Published online: 22 April 2017

(C) The Author(s) 2017. This article is an open access publication

\begin{abstract}
Background It is well known that atrophic renal changes are associated with chronic kidney disease (CKD) progression, but conventional diagnostic imaging methods such as noncontrast-enhanced computed tomography and magnetic resonance imaging (MRI) have been insufficient for precisely assessing kidney function because they cannot clearly distinguish between the medulla and cortex. Hence, here we used noncontrast-enhanced steady-state free precession (SSFP) MRI with a spatially selective inversion recovery (IR) pulse to improve visibility for renal corticomedullary differentiation and evaluated the association between morphological parameters and kidney function in patients with CKD.

Methods Kidney corticomedullary contrast ratio, cortical and medullary areas, and minimal cortical thickness of 107 patients with CKD G1-G5 were measured using SSFP MRI with a spatially selective IR pulse and the association between these morphological parameters and kidney function were evaluated.

Results Corticomedullary contrast ratio was significantly improved on SSFP MRI compared with conventional inphase T1-weighted gradient-echo MRI and positively correlated with estimated glomerular filtration ratio (eGFR), raw eGFR, and 24-h creatinine clearance. The medullary and cortical areas and minimal cortical thickness also
\end{abstract}

Yoshikatsu Kaneko

kanekoy@med.niigata-u.ac.jp

1 Division of Clinical Nephrology and Rheumatology, Niigata University Graduate School of Medical and Dental Sciences, 1-757 Asahimachi-dori, Niigata 951-8510, Japan

2 Tachikawa General Hospital, 561-1 Azayachi, Jojomachi, Nagaoka, Niigata 940-8621, Japan positively correlated with those of kidney functional markers and the age. In patients with CKD and diabetes mellitus (DM), the correlation coefficients between raw eGFR and morphological parameters were higher than those in patients without DM, while minimal cortical thickness was larger in CKD patients with DM with a raw eGFR $\geq 45 \mathrm{~mL} / \mathrm{min}$.

Conclusion Kidney morphological parameters measured with SSFP MRI were clearly correlated with kidney function in patients with $\mathrm{CKD}$, including those with advanced kidney dysfunction.

Keywords SSFP MRI - Cortical thickness - Cortical area · Medullary area

\section{Introduction}

In chronic kidney disease (CKD), kidney dysfunction progression correlates with reduced bipolar kidney length, kidney volume, and cortical thickness [1]. Kidney biopsy is an effective method for obtaining pathological information and evaluating CKD progression; however, percutaneous kidney biopsy is rather contraindicated for patients with advanced CKD and an atrophic renal cortex. Therefore, non-invasive quantitative and qualitative evaluations such as ultrasonography (US), computed tomography (CT), or magnetic resonance imaging (MRI) have long been attempted to assess morphological changes in the diseased kidney and estimate the etiology, duration, and reversibility of kidney disease.

Among these diagnostic imaging methods, US has often been used in the initial evaluation of CKD patients for its convenience. Measurement of the renal cortical thickness using US was reported to be valuable for estimating 
residual kidney function in CKD patients and predicting their prognosis [2]. However, its interobserver reproducibility was not guaranteed and its reproducibility for cortical thickness measurements was not satisfactory [3]. Although contrast-enhanced CT is considered an accurate method for measuring renal morphological parameters including renal cortical thickness, its routine use for this purpose is limited due to the potential nephrotoxicity of contrast agents.

Noncontrast MRI was previously considered insufficient to distinguish between the renal cortex and medulla, especially in patients with kidney dysfunction because of an increased $\mathrm{T} 1$ relaxation time of the cortex accompanied with decreased kidney function [4, 5], so the evaluation of corticomedullary differentiation required dynamic contrast-enhanced MRI. However, the risk of nephrogenic systemic fibrosis caused by contrast agent use could not be neglected [6]. Recently, noncontrast-enhanced steady-state free precession (SSFP) MRI with a spatially selective inversion recovery (IR) pulse was reported to improve the resolution of renal corticomedullary differentiation in patients without a history of renal disease [7] and in those with CKD [8]. Noda et al. reported a significant association between minimal renal cortical thickness and estimated glomerular filtration rate (eGFR) in patients with CKD using this method. However, the mean eGFR of the participants in the previous report was $79.7 \mathrm{~mL} / \mathrm{min} / 1.73 \mathrm{~m}^{2}$ (range $36.1-125.9 \mathrm{~mL} / \mathrm{min} / 1.73 \mathrm{~m}^{2}$ ), while only 16 of 65 participants had an eGFR $<60.0 \mathrm{~mL} / \mathrm{min} / 1.73 \mathrm{~m}^{2}$ [8]. The usefulness of this method has not yet been proven in patients with more advanced CKD stages, such as CKD G4 and G5.

Therefore, the purposes of the present study were to assess whether noncontrast-enhanced SSFP MRI with a spatially selective IR pulse improved the visibility of renal corticomedullary differentiation in patients with CKD including those with advanced stages and to investigate the correlation between kidney function and segmental areas of the kidney compartments.

\section{Materials and methods}

\section{Subjects}

In our retrospective study, we identified 107 patients diagnosed with CKD who underwent noncontrast-enhanced SSFP MRI with spatially selective IR pulse at Tachikawa General Hospital between February 2015 and July 2016. Clinical evaluations were conducted within a week after MRI examination. Patients were excluded from the study if they had a single kidney, a large solid/cystic lesion in the kidney, autosomal dominant polycystic kidney disease, acute kidney injury, unacceptable image quality, or insufficient medical information. Patients with a rotated kidney were also excluded because of the possible incorrectness of measuring morphological parameters. The Institutional Review Board of Tachikawa General Hospital approved this retrospective study (approval number 12000056) and the patients provided written informed consent.

\section{Definition}

CKD was defined as an eGFR $<60 \mathrm{~mL} / \mathrm{min} / 1.73 \mathrm{~m}^{2}$ and/ or the presence of proteinuria (urine protein $\geq 0.15 \mathrm{~g} / \mathrm{gCr}$ or urine albumin $\geq 30 \mathrm{mg} / \mathrm{gCr}$ ), and these abnormalities of the kidney were present for more than 3 months and were staged according to the 2012 Kidney Disease: Improving Global Outcomes (KDIGO) guidelines [9, 10]. The eGFR values were calculated using the modification of diet in renal disease formula for Japanese patients: eGFR $(\mathrm{mL} /$ $\left.\mathrm{min} / 1.73 \mathrm{~m}^{2}\right)=194 \times$ [serum creatinine $\quad(\mathrm{mg} /$ $\mathrm{dL})]^{-1.094} \times[\text { age }(\text { years })]^{-0.287} \times 0.739$ (if female) [11]. Body surface area (BSA) was calculated according to the DuBois and DuBois formula [12]. Raw eGFR was calculated using the following equation: raw eGFR $(\mathrm{mL} /$ $\min )=\mathrm{eGFR} \times \mathrm{BSA} / 1.73$. The diagnosis of diabetes mellitus (DM) was based on American Diabetes Association criteria [13] or on the basis of a history of type $2 \mathrm{DM}$ under dietary intervention or the use of hypoglycemic agents. Patients who received anti-hypertensive agents were defined as those treated for more than 6 months.

\section{Imaging technique}

All MRI examinations for SSFP images were performed with a 1.5-T unit (Vantage Atlas MRT-2003; Toshiba Medical Systems, Tokyo, Japan) using the Atlas SPEE$\mathrm{DER}^{\mathrm{TM}}$ body coil. The procedure for the MRI imaging was described in the previous study by Kanki et al. [7]. Imaging parameters of the SSFP sequence were as follows: repetition time/echo time $(\mathrm{TR} / \mathrm{TE})=4.2 / 2.1 \mathrm{~ms}$; number of acquisitions $=1 ;$ parallel imaging factor $=2 ;$ flip angle $=90^{\circ}$; receiver bandwidth $=977 \mathrm{~Hz} /$ pixel; slice thickness $=7 \mathrm{~mm}$; field of view $=400 \times 400 \mathrm{~mm}$; acquisition matrix $=256 \times 256$. Noncontrast-enhanced SSFP MRI of the kidney with a spatially selective IR pulse was performed using identical imaging parameters during a single breath hold. A spatially selective IR pulse with a thickness of $130 \mathrm{~mm}$ was placed on both kidneys. To measure and compare each parameter of kidney imaging under the same conditions, $1400 \mathrm{~ms}$ was defined as a standard TI by reference to a previous report. In addition, in-phase (IP) T1-weighted gradient-echo MR images were obtained to compare the visibility of corticomedullary 
differentiation as well as the corticomedullary contrast ratio of SSFP MRI with a spatially selective IR pulse. Imaging parameters of the IP sequence were as follows: $\mathrm{TR} / \mathrm{TE}=240 / 4.8 \mathrm{~ms}$; number of acquisitions $=1$; flip angle $=90^{\circ}$; receiver bandwidth $=488 \mathrm{~Hz} /$ pixel; slice thickness $=7 \mathrm{~mm}$; field of view $=400 \times 400 \mathrm{~mm}$; acquisition matrix $=320 \times 192$ [7]. For comparison with conventional IP images at 3.0-T unit, MRI examinations were conducted with GE SIGNA ${ }^{\mathrm{TM}}$ Pioneer 3.0 T scanner (GE Healthcare, Piscataway, NJ, USA) using TDI Anterior Array coil and TDI Posterior Array coil.

\section{Data analyses}

All images were reviewed on a clinical picture archiving and communication system workstation monitor (Rapideye Core; Toshiba Medical Systems) by two physicians blinded to the subjects' clinical information. The physicians selected the larger of the right or left kidney as the measuring object and drew operator-defined regions of interest (ROI) within the cortex and medulla in the target kidney to measure the signal intensity (SI) values of each segment by consensus. ROI measurements were also performed on the IP images. The corticomedullary contrast ratio was calculated from SI values of the renal cortex $\left(\mathrm{SI}_{\text {cortex }}\right)$ and renal medulla ( $\left.\mathrm{SI}_{\text {medulla }}\right)$ as $\left(\mathrm{SI}_{\text {cortex }} / \mathrm{SI}_{\text {medulla }}\right)$. Additionally, the physicians measured and recorded the minimal cortical thicknesses and coronal sectional areas of the cortex and medulla as shown in Fig. 1.

\section{Statistical analyses}

Corticomedullary contrast ratios between SSFP and IP images were compared using the Wilcoxon signed-rank test. The Pearson product-moment correlation coefficient was used to investigate the correlation between two parameters in each case. Simple associations were performed using unpaired $t$ tests or appropriate non-parametric tests for continuous variables and Chi-square tests for categorical variables. The interobserver agreements in relation to morphological parameters were verified by intraclass correlation coefficient (ICC). Value of $P<0.05$ was considered statistically significant.

\section{Results}

\section{Characteristics of the study subjects and morphological variables of the kidney}

A total of 107 patients diagnosed with CKD were enrolled in this study. Table 1 presents the background profiles of the entire study population. The median age was 64 years

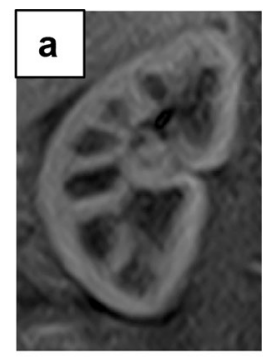

SSFP image (CKDG1)

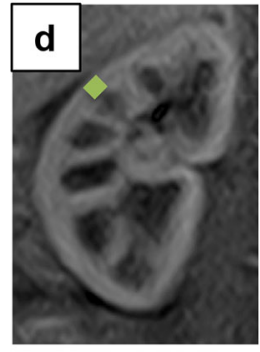

Minimal thickness

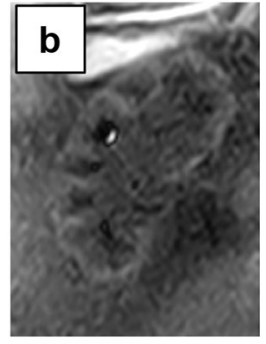

SSFP image (CKDG5)

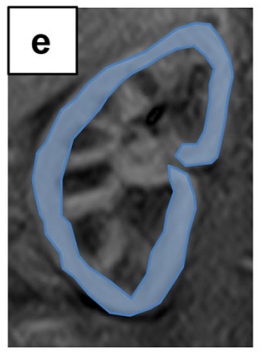

Renal cortical area

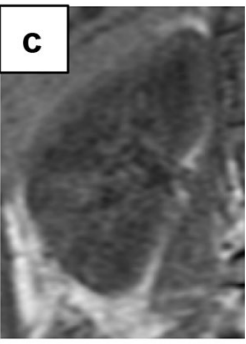

IP image (CKDG1)

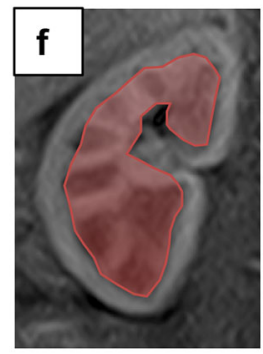

Renal medullary area
Fig. 1 Representative coronal kidney images of noncontrast-enhanced steady-state free precession (SSFP) magnetic resonance imaging (MRI) with spatially selective inversion recovery pulse at 1.5-T unit $(\mathbf{a}, \mathbf{b}, \mathbf{d}-\mathbf{f})$ or conventional in-phase MRI at 3.0-T unit (c). a An SSFP image of a CKD G1 patient. b An SSFP image of a CKD G5 patient. c A conventional in-phase image of a CKD G1 patient. Minimal cortical thickness, cortical area, and medullary area are shown as a yellow band (d), blue area (e), and red area (f), respectively

and $60.7 \%$ of the patients were men. The mean eGFR, raw eGFR, and 24-h creatinine clearance (Ccr) were $51.1 \pm 22.7 \mathrm{~mL} / \mathrm{min} / 1.73 \mathrm{~m}^{2}$ (range 8.4-130.2), $53.7 \pm$ $25.4 \mathrm{~mL} / \mathrm{min}$ (range 7.6-118.6), and $67.6 \pm 31.7 \mathrm{~mL} / \mathrm{min}$ (range 4.0-179.0), respectively. The numbers and percentages of patients with each CKD stage were as follows: CKD G1, four (3.7\%); G2, 35 (32.7\%); G3, 48 (44.9\%); G4, 13 (12.1\%); G5, seven (6.5\%). Anti-hypertensive agents were used in $93(86.9 \%)$ patients, and 73 patients $(68.2 \%)$ were treated with renin-angiotensin system (RAS) inhibitors, whereas 14 patients $(13.1 \%)$ were treated with diuretics. Renal corticomedullary differentiation was clearly depicted in SSFP images using standard TI, but not in conventional IP images at even 3.0-T unit MRI (Fig. 1). The mean corticomedullary contrast ratio was higher in SSFP images than in IP images with some exceptional cases (Fig. 2), and the corticomedullary contrast ratio in SSFP images positively correlated with all of the three kidney functional parameters (Fig. 3). Then three morphological variables, minimal cortical thickness, cortical area, and medullary area of all patients were subjected to the validation study of interobserver reproducibility, and each ICC demonstrated a good correlation (Table 2). Average values of the measured kidney morphological parameters are shown in Table 3 . 
Table 1 Clinical characteristics of the patients with CKD $(n=107)$

\begin{tabular}{|c|c|}
\hline Male sex $[n(\%)]$ & $65(60.7)$ \\
\hline Age (years; mean $\pm \mathrm{SD}$, range) & $64 \pm 17,18-88$ \\
\hline Height $(\mathrm{m} ;$ mean $\pm \mathrm{SD})$ & $1.61 \pm 0.10$ \\
\hline Weight $(\mathrm{kg}$; mean $\pm \mathrm{SD})$ & $62.9 \pm 14.9$ \\
\hline $\mathrm{BMI}($ mean $\pm \mathrm{SD})$ & $24.2 \pm 4.2$ \\
\hline $\mathrm{BSA}\left(\mathrm{m}^{2} ;\right.$ mean $\left.\pm \mathrm{SD}\right)$ & $1.79 \pm 0.25$ \\
\hline BUN [mg/dL; median (IQR)] & $17.0(13.6-22.6)$ \\
\hline Creatinine $[\mathrm{mg} / \mathrm{dL}$; median (IQR)] & $1.02(0.84-1.53)$ \\
\hline $\mathrm{eGFR}\left(\mathrm{mL} / \mathrm{min} / 1.73 \mathrm{~m}^{2} ;\right.$ mean $\pm \mathrm{SD}$, range $)$ & $51.1 \pm 22.7,8.4-130.2$ \\
\hline Raw eGFR (mL/min; mean $\pm \mathrm{SD}$, range) & $53.7 \pm 25.4,7.6-118.6$ \\
\hline $24 \mathrm{~h}$ Ccr $(\mathrm{mL} / \mathrm{min}$; mean $\pm \mathrm{SD}$, range $)$ & $67.6 \pm 31.7,4.0-179.0$ \\
\hline Urinary protein [mg/day; median (IQR)] & $182(52-865)$ \\
\hline \multicolumn{2}{|l|}{ CKD stage $[n(\%)]$} \\
\hline G1 & $4(3.7)$ \\
\hline $\mathrm{G} 2$ & $35(32.7)$ \\
\hline G3 & $48(44.9)$ \\
\hline G4 & $13(12.1)$ \\
\hline G5 & $7(6.5)$ \\
\hline HbA1c $(\% ;$ mean $\pm \mathrm{SD})$ & $5.8 \pm 0.7$ \\
\hline Anti-hypertensive agents $[n(\%)]$ & $93(86.9)$ \\
\hline RAS inhibitor $[n(\%)]$ & $73(68.2)$ \\
\hline Calcium channel blockers $[n(\%)]$ & $49(45.8)$ \\
\hline Diuretics $[n(\%)]$ & $14(13.1)$ \\
\hline Beta-blockers $[n(\%)]$ & $16(15.0)$ \\
\hline
\end{tabular}

$C K D$ chronic kidney disease, $B M I$ body mass index, BSA body surface area, $B U N$ blood urea nitrogen, $e G F R$ estimated glomerular filtration rate, $C c r$ creatinine clearance, $R A S$ renin-angiotensin system

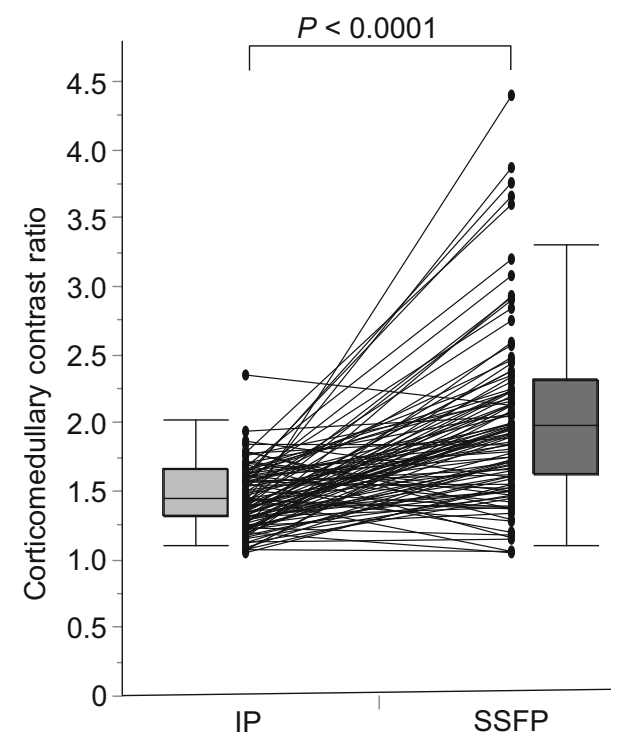

Fig. 2 Comparison of corticomedullary contrast ratio. Mean corticomedullary contrast ratio of the patients with chronic kidney disease $(n=107)$ was significantly higher in steady-state free precession (SSFP) images than in in-phase (IP) images

\section{Correlation between kidney morphological parameters and function in patients with CKD}

The correlations between kidney morphological parameters and kidney functional markers were assessed by analysis of variance. All of the three parameters were significantly correlated with eGFR, raw eGFR, and 24-h Ccr. Among these kidney morphological parameters, cortical area demonstrated the highest correlation coefficients with all three kidney functional markers. Regarding kidney functional markers, raw eGFR exhibited the highest correlation coefficients with all three kidney morphological parameters (Fig. 4), suggesting that the cortical area is the most efficient parameter for estimating raw eGFR in patients with CKD. Three patients had a unilateral atrophic kidney with volume reduction and a unilateral renal artery stenosis on the same side. In these cases, no significant correlation between minimal cortical thickness of the atrophic kidney and kidney function was observed.

We also analyzed the correlations between daily urinary protein level and each kidney morphological parameter since urinary protein level reflects kidney disease severity. However, no significant correlations were observed among them (Fig. 5). 

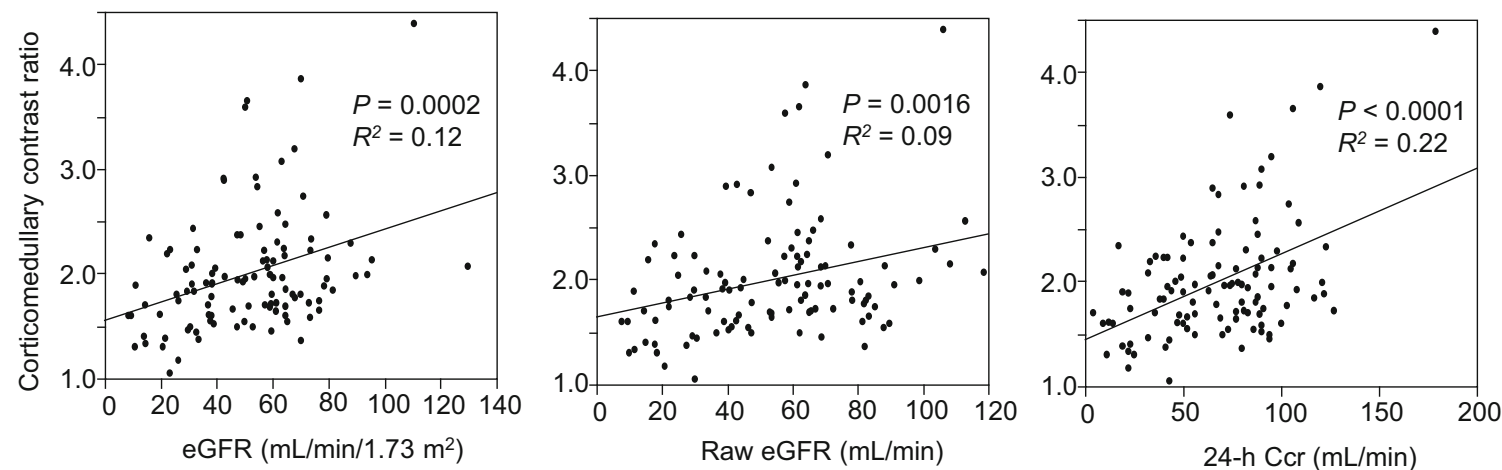

Fig. 3 Correlation between corticomedullary contrast ratio and estimated glomerular filtration rate (eGFR), raw eGFR, or 24-h creatinine clearance $(\mathrm{Ccr})$. The corticomedullary contrast ratio was positively correlated with all three markers of kidney function

Table 2 Interobserver reproducibility in the measurement of kidney morphological parameters

\begin{tabular}{llll}
\hline & ICC & Interval for the ICC of 95\% & $P$ value \\
\hline Minimal cortical thickness & 0.866 & $0.809-0.906$ & $<0.0001$ \\
Cortical area & 0.988 & $0.982-0.992$ & $<0.0001$ \\
Medullary area & 0.941 & $0.915-0.956$ & $<0.0001$ \\
\hline
\end{tabular}

ICC intraclass correlation coefficient
Table 3 Measured kidney morphological parameters

\begin{tabular}{ll}
\hline Cortical thickness $(\mathrm{mm} ;$ mean $\pm \mathrm{SD})$ & \\
Maximal & $7.5 \pm 1.9$ \\
Minimal & $5.3 \pm 1.7$ \\
Coronal sectional area $\left(\mathrm{cm}^{2} ;\right.$ mean $\left.\pm \mathrm{SD}\right)$ & \\
Cortex & $12.88 \pm 4.21$ \\
Medulla & $17.24 \pm 5.65$ \\
Entire kidney & $30.11 \pm 8.51$ \\
\hline
\end{tabular}

\section{Correlation between kidney morphological parameters and age in patients with CKD}

Then we analyzed the correlation between age and each morphological parameter, because GFR is progressively decreased by aging $[14,15]$ and age-related reduction of eGFR could affect the morphological parameters. Figure 6 shows that negative correlations were observed between age and each morphological parameter. We also estimated original raw eGFR from each morphological parameter using regression line in Fig. 4, and the predicted raw eGFR also significantly correlated with the age.

\section{Correlation between kidney morphological parameters and function in CKD patients with or without DM}

Finally, we divided the CKD patients into DM $(n=18)$ and non-DM $(n=89)$ groups to evaluate the association between CKD etiology and kidney morphological parameters. Table 4 represents the background profiles of the two groups. Patients with DM were older and had larger body weight, larger body mass index, and larger BSA. HbA1c was also higher in patients with DM. RAS inhibitors were administered to significantly more patients without DM, and larger proportion of patients with DM was treated with diuretics and beta-blockers. Otherwise, no clinical characteristics differed between the two groups. We adopted raw eGFR as a kidney functional marker to evaluate the differences in correlation with morphological parameters between the patients with and without DM since raw eGFR had the highest correlation coefficient with each kidney morphological parameter (Fig. 4). Figure 7 shows that in patients with DM, all three morphological parameters demonstrated higher correlation coefficients with raw eGFR than those in patients without DM. Furthermore, minimal cortical thickness was significantly greater in patients with DM and a raw eGFR $\geq 45 \mathrm{~mL} / \mathrm{min}$ (Fig. 8), indicating that the atrophic change in the kidney cortex was milder in the earlier stage of CKD in patients with DM.

\section{Discussion}

Our study revealed that renal corticomedullary differentiation was more clearly depicted in SSFP MRI with a spatially selective IR pulse than in conventional IP images, even in patients with CKD G4 and G5. This new imaging technique enabled us to measure the thickness or area of 

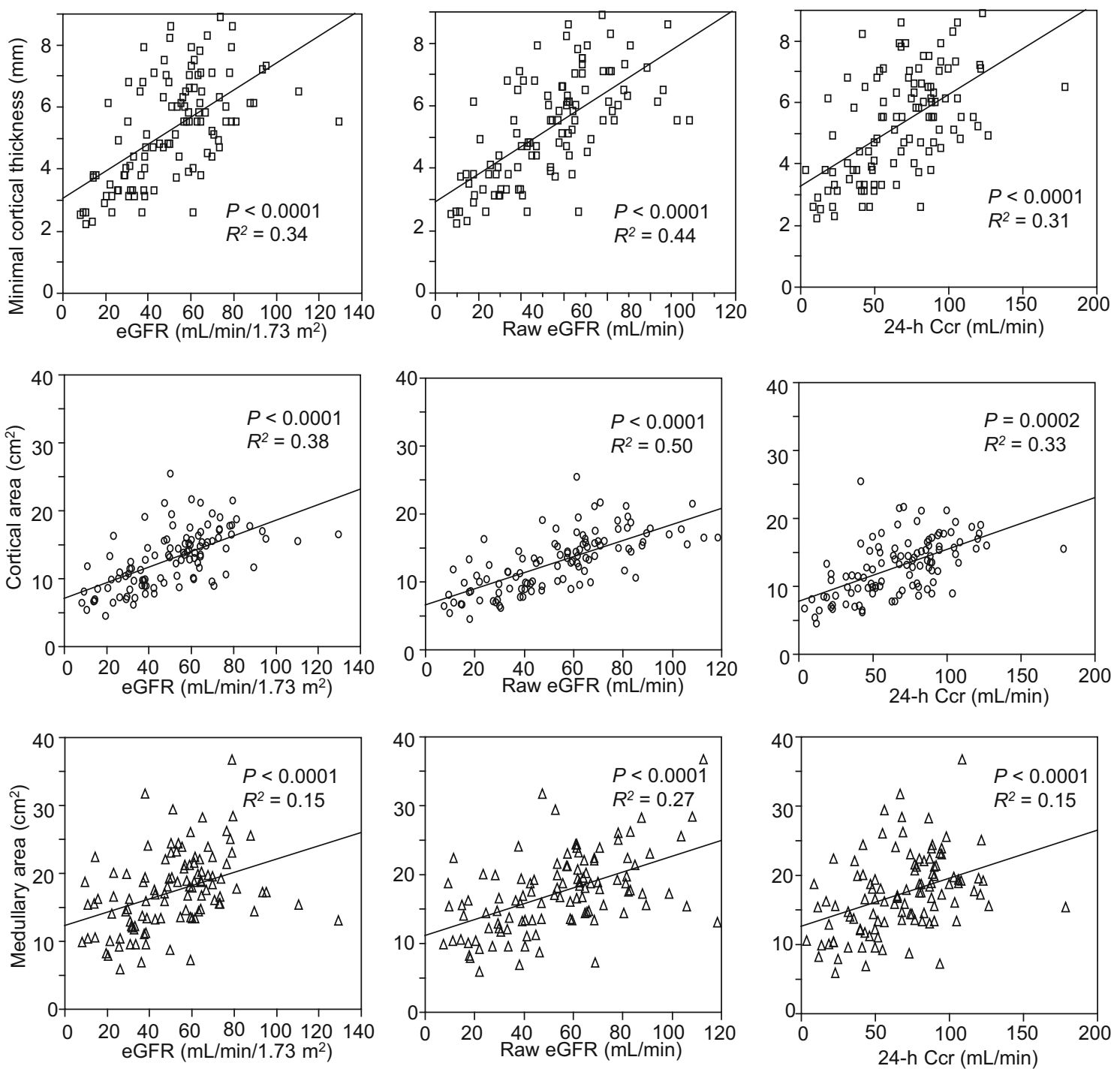

Fig. 4 Correlation between kidney function and minimal cortical thickness (upper), cortical area (middle), or medullary area (lower). All three morphological parameters were positively correlated with estimated glomerular filtration rate (eGFR), raw eGFR, or 24-h creatinine clearance $(\mathrm{Ccr})$
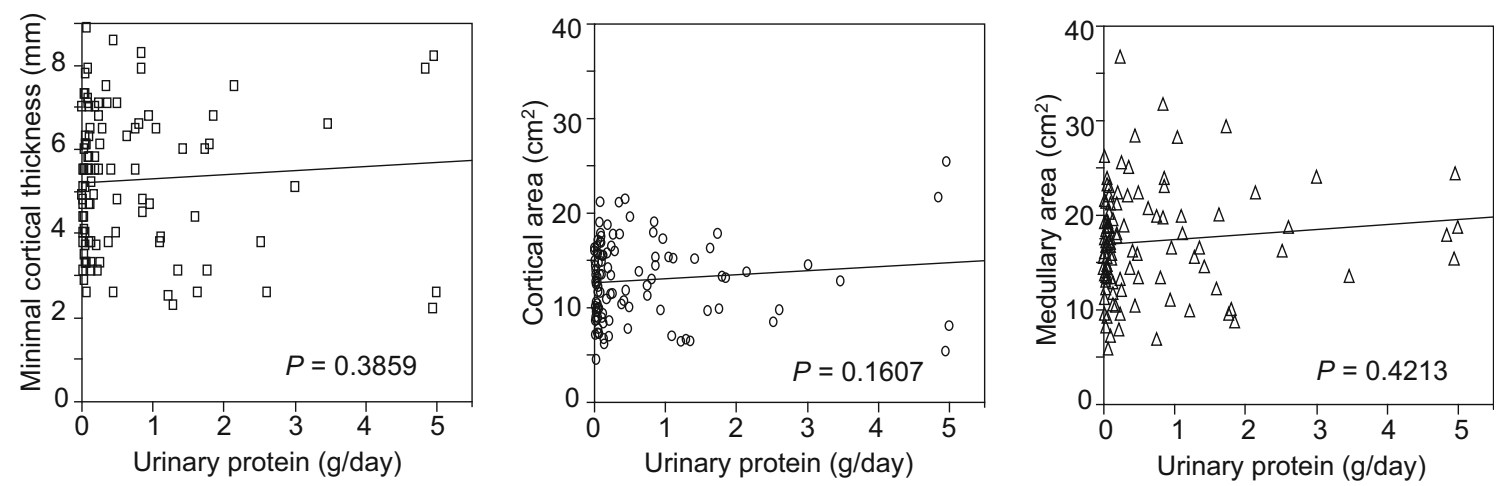

Fig. 5 Correlation between 24-h urinary protein amount and minimal cortical thickness, cortical area, or medullary area. There were no significant correlations between 24-h urinary protein amount and kidney morphological parameters 

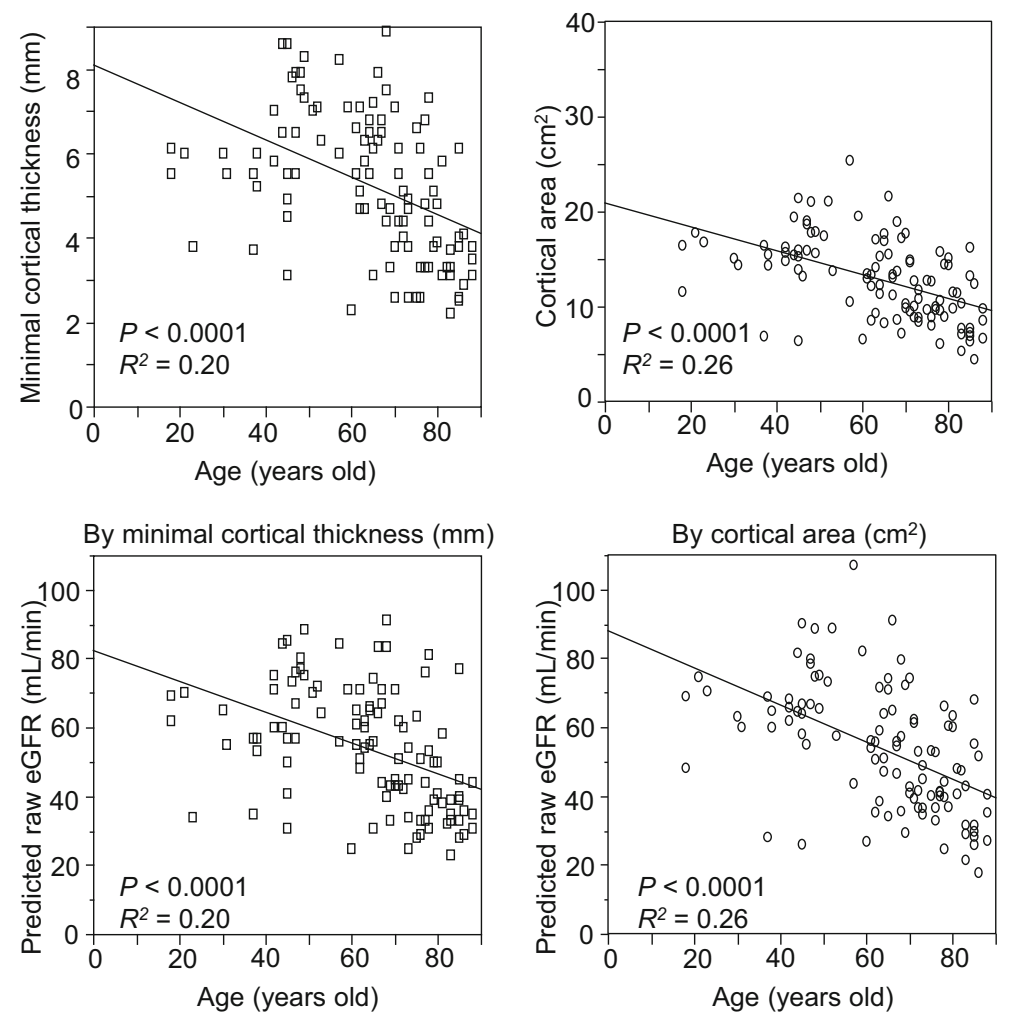
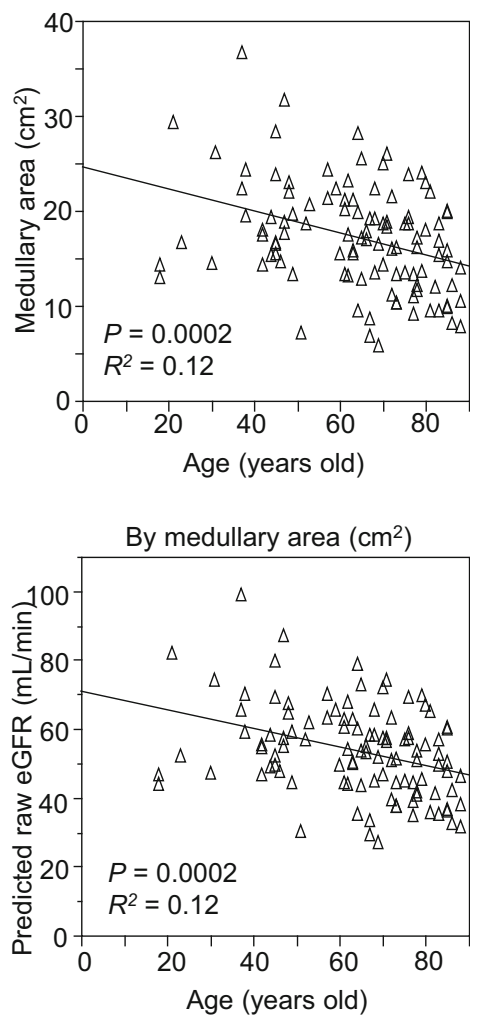

Fig. 6 Correlation between age and morphological parameters. All of three morphological parameters, minimal cortical thickness, cortical area, and medullary area (upper) were negatively correlated with the age of the CKD patients. Predicted raw eGFR based on each morphological parameter using its peculiar regression line in Fig. 4 also significantly correlated with the age (lower). Predicted raw eGFR

the each renal segment without contrast agents in patients with CKD and evaluate renal cortex atrophy.

It has been reported that minimal renal cortical thickness has a positive correlation with renal function because nephrons are mainly located in the renal cortex [16], but the corticomedullary boundary in unenhanced MRI reportedly becomes ambiguous in patients with advanced renal insufficiency, making it difficult to properly measure renal cortical thickness $[4,5]$. MRI with the time-spatial labeling inversion pulse method was originally developed to investigate blood flow to ROI by selectively placing the IR pulse and saturating blood spins in the vessels and background [7]. Kanki et al. reported that they applied the SSFP MRI in 20 patients and revealed the improved visibility of renal corticomedullary differentiation by accentuating the differences in $\mathrm{T} 1$ values between the renal cortex and medulla without using contrast agents. In SSFP MRI with time-special labeling inversion pulse obtained with optimal TI, protons in the renal cortex with shorter T1 values are substantially magnetized (recovered) while the longitudinal magnetization of protons in the renal medulla with longer $\mathrm{T} 1$ values is nulled. Thus, the renal cortex is was calculated as follows: predicted raw eGFR by minimal cortical thickness $\quad(\mathrm{mL} / \mathrm{min})=10.06 \times$ minimal cortical thickness $(\mathrm{mm})+0.728$ (left); predicted raw eGFR by cortical area $(\mathrm{mL} /$ min) $=4.3 \times$ cortical area $\left(\mathrm{cm}^{2}\right)-1.363$ (middle); predicted raw eGFR by medullary area $(\mathrm{mL} / \mathrm{min})=2.3 \times$ medullary area $\left(\mathrm{cm}^{2}\right)+13.42($ right $)$

shown as bright, while the renal medulla is observed as dark, resulting in the better visualization of corticomedullary differentiation [7]. The same research group reported in a series of papers that visualization of the corticomedullary differentiation was significantly better in SSFP images than that on fast asymmetric spin echo images, another method used for noncontrast-enhanced MR angiography [17]. They also reported that this SSFP imaging method was also useful without the influence of aging if optimal TI values for the best visualization were adopted [18]. In the present study, we revealed that corticomedullary contrast ratio was significantly improved by SSFP MRI, even though it was smaller in some cases (Fig. 2). Ideally, optimal TI for each case should be determined for the best visualization of renal corticomedullary junction [7, 18]. In our study, however, we standardized TI for measurement as $1400 \mathrm{~ms}$ to compare the corticomedullary contrast ratios under the same condition. Therefore, medulla signals would have been inappropriately higher in some cases.

We also confirmed that morphological parameters demonstrated negative correlation with age (Fig. 6). In a 
Table 4 Clinical characteristics of the patients with CKD with or without diabetes mellitus

\begin{tabular}{|c|c|c|c|}
\hline Characteristic & $\mathrm{DM}(n=18)$ & Non-DM $(n=89)$ & $P$ value \\
\hline Male sex $[n(\%)]$ & $14(77.8)$ & $51(57.3)$ & 0.1047 \\
\hline Age (years; mean $\pm \mathrm{SD}$ ) & $67 \pm 15$ & $63 \pm 18$ & 0.0019 \\
\hline Height $(m ;$ mean \pm SD) & $1.60 \pm 0.13$ & $1.60 \pm 0.01$ & 0.6772 \\
\hline Weight $(\mathrm{kg} ;$ mean $\pm \mathrm{SD})$ & $71.0 \pm 20.0$ & $61.3 \pm 13.2$ & 0.0113 \\
\hline BMI (mean \pm SD) & $24.2 \pm 4.2$ & $23.7 \pm 3.8$ & 0.0026 \\
\hline $\mathrm{BSA}\left(\mathrm{m}^{2} ;\right.$ mean $\left.\pm \mathrm{SD}\right)$ & $1.91 \pm 0.33$ & $1.77 \pm 0.23$ & 0.0118 \\
\hline BUN [mg/dL; median (IQR)] & $18.1(13.5-33.1)$ & $17.0(13.8-21.0)$ & 0.6320 \\
\hline Cre $[\mathrm{mg} / \mathrm{dL} ;$ median (IQR)] & $1.08(0.82-2.42)$ & $1.02(0.87-1.45)$ & 0.4946 \\
\hline eGFR $\left(\mathrm{mL} / \mathrm{min} / 1.73 \mathrm{~m}^{2} ;\right.$ mean $\left.\pm \mathrm{SD}\right)$ & $50.0 \pm 27.9$ & $51.3 \pm 21.6$ & 0.8225 \\
\hline Raw eGFR (mL/min; mean $\pm \mathrm{SD})$ & $57.2 \pm 36.0$ & $53.0 \pm 22.9$ & 0.5164 \\
\hline 24-h Ccr (mL/min; mean \pm SD) & $61.5 \pm 38.6$ & $68.8 \pm 30.2$ & 0.3762 \\
\hline Urinary protein [mg/day; median (IQR)] & $458(136-1610)$ & $126(48-829)$ & 0.2061 \\
\hline CKD stage $[n(\%)]$ & & & 0.0699 \\
\hline G1 & $0(0.0)$ & $4(4.5)$ & \\
\hline $\mathrm{G} 2$ & $9(50.0)$ & $26(29.2)$ & \\
\hline G3 & $4(22.2)$ & $44(49.4)$ & \\
\hline G4 & $2(11.1)$ & $11(12.4)$ & \\
\hline G5 & $3(16.7)$ & $4(4.5)$ & \\
\hline HbA1c $(\% ;$ mean $\pm \mathrm{SD})$ & $7.1 \pm 0.7$ & $5.6 \pm 0.3$ & $<0.0001$ \\
\hline Anti-hypertensive agents $[n(\%)]$ & $14(77.8)$ & $79(88.8)$ & 0.2357 \\
\hline RAS inhibitors $[n(\%)]$ & $8(44.4)$ & $65(73.0)$ & 0.0214 \\
\hline Calcium channel blockers $[n(\%)]$ & $10(55.6)$ & $39(43.8)$ & 0.3629 \\
\hline Diuretics $[n(\%)]$ & $6(33.3)$ & $8(9.0)$ & 0.0120 \\
\hline Beta-blockers [n (\%)] & $8(44.4)$ & $8(9.0)$ & 0.0006 \\
\hline
\end{tabular}

$C K D$ chronic kidney disease, $B M I$ body mass index, $B S A$ body surface area, $B U N$ blood urea nitrogen, eGFR estimated glomerular filtration rate, $C c r$ creatinine clearance, $R A S$ renin-angiotensin system previous study using contrast-enhanced CT, kidney cortical volume alone, but not medullary, was reported to correlate negatively with the age [19]. In the other study, no significant correlation was observed between age and renal cortical thickness measured by SSFP MRI [18]. In these previous studies, patients with decreased kidney function were not included. We surmise that correlation with age and atrophic change of kidney could be confirmed when aged patients with advanced kidney dysfunction were included. Moreover, predicted raw eGFR based on each morphological parameter also significantly correlated with the age, suggesting that SSFP MRI could be substitutable for GFR. We are sure that the present study is complementary to the series of previous reports regarding the effectiveness of SSFP MRI for the more precise evaluation of kidney morphological parameters even in patients with advanced CKD.

On the contrary, the measured morphological parameters had no significant correlations with the urine protein level. This fact may indicate that decreased morphological values reflect chronic atrophic changes but not ongoing glomerular or tubular damage expressed as elevated urine protein level. Morphological changes caused by tubular atrophy and following tubulointerstitial fibrosis would have been valuable indicators for the assessment of residual kidney function and reversibility in progressive chronic kidney disease [20]. We are sure that this morphological assessment would be useful for assessing kidney dysfunction progression.

We also compared the morphological parameters of CKD patients with and without DM and demonstrated that kidney morphological parameters correlated with raw eGFR better in the CKD patients with DM than in those without DM (Fig. 7). We surmised that the kidney atrophic changes of the patients without DM were not homogenous because of the heterogeneity of the etiology of CKD in this group. We also confirmed that minimal cortical thickness was greater in patients with DM with a raw eGFR $\geq 45 \mathrm{~mL} / \mathrm{min}$. It has been reported that at the onset of DM, a subset of diabetic patients undergo an increase in GFR caused by glomerular hyperfiltration and kidney growth due to accelerated reabsorption in proximal tubule $[21,22]$. Our data were in line with these findings; however, we could not exclude the influence of diuretics, which 
Patients with DM $(\mathrm{n}=18)$

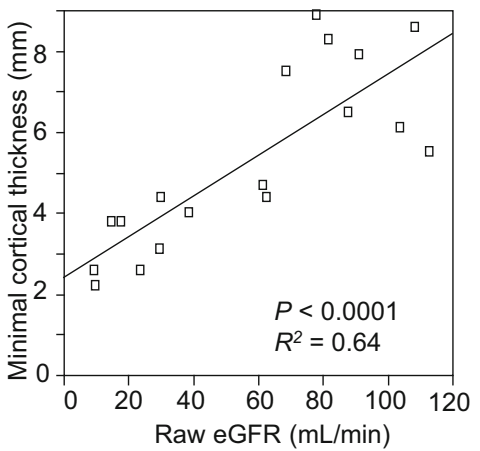

Patients without DM $(\mathrm{n}=89)$

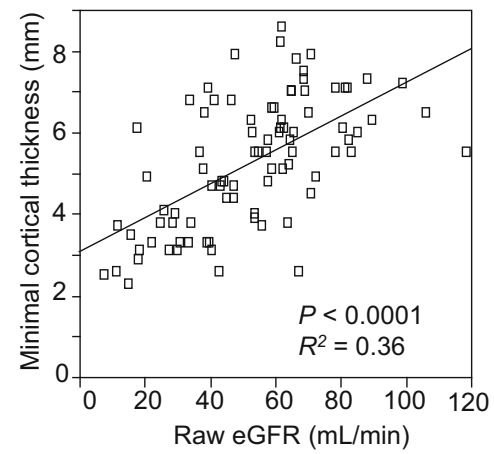

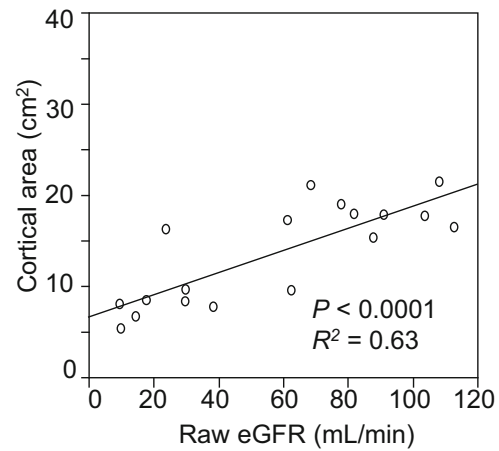
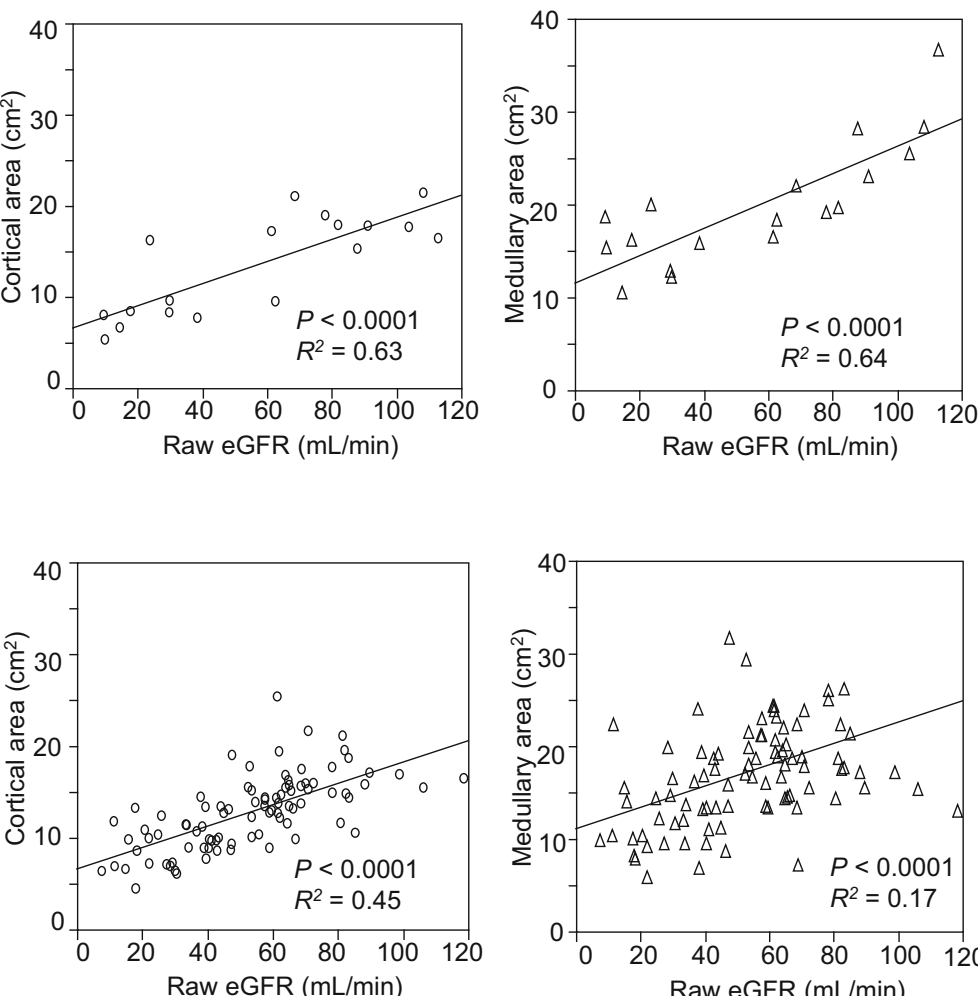

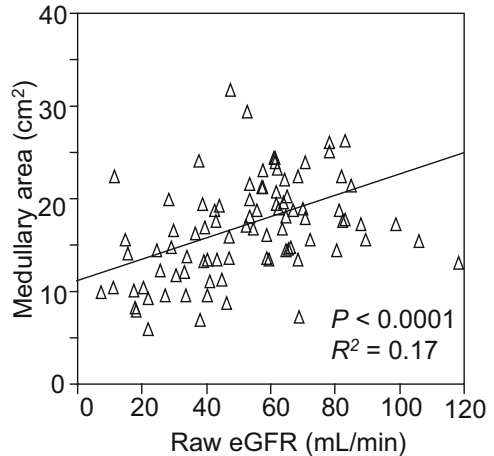

Fig. 7 Correlation between raw estimated glomerular filtration rate (eGFR) and minimal cortical thickness, cortical area, or medullary area in patients with chronic kidney disease (CKD) with diabetes
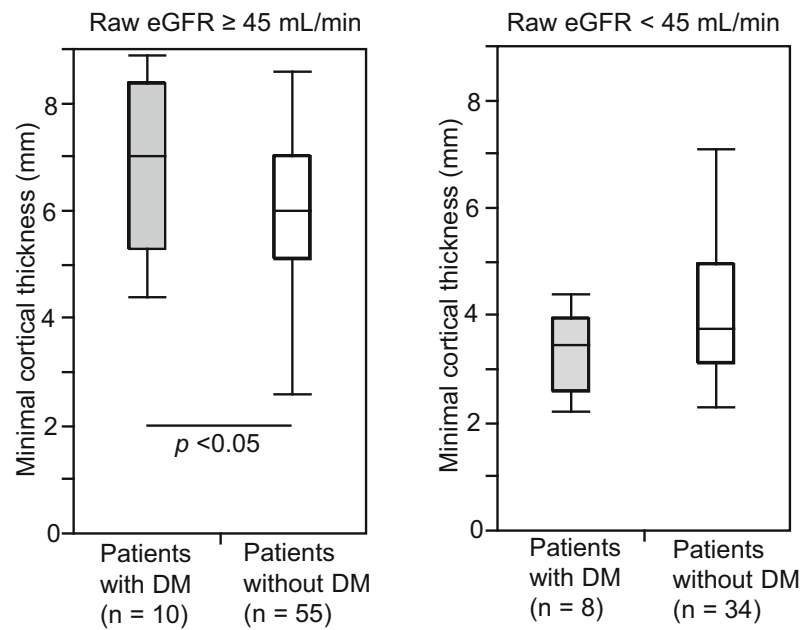

Fig. 8 Comparison of minimal cortical thickness between patients with chronic kidney disease (CKD) with or without diabetes mellitus (DM). The patients with CKD were divided into two groups, those with a raw estimated glomerular filtration rate (eGFR) $\geq 45 \mathrm{~mL} / \mathrm{min}$ and those with a raw eGFR $<45 \mathrm{~mL} / \mathrm{min}$, and minimal cortical thicknesses were compared between patients with CKD and DM and those with CKD but without DM. Values are shown as mean $\pm \mathrm{SD}$ mellitus (DM) $(n=18)$ or without DM $(n=89)$. All three kidney morphological parameters showed higher correlation coefficients with raw eGFR in CKD patients with DM than in those without DM

were administered in significantly larger proportion of the patients with DM. Diuresis could have elevated serum creatinine level, resulting in underestimation of eGFR in patients with preserved kidney size and function.

In the present study, raw eGFR demonstrated the highest correlation coefficient with each of the three kidney functional parameters evaluated in the study. Twenty-four-hour Ccr usually overestimates kidney function due to the tubular secretion of creatinine, especially in patients with advanced CKD and decreased glomerular filtration [23]. The eGFR value is normalized for BSA, but considering that renal cortical volume is positively correlated with BSA and GFR [18, 19, 24], raw eGFR would represent morphological kidney changes in CKD more accurately than eGFR normalized for BSA.

The limitation of this study was that the categorization of patients with CKD with or without DM was not based on the definite diagnosis by kidney biopsy but was clinically different by the presence of DM. The definite histopathological diagnosis by kidney biopsy would be essential to reliably utilize these kidney morphological parameters for the differential diagnosis of the CKD origin using noninvasive SSFP MRI. Another limitation of this study was that this study was performed with 1.5-T unit MRI. Even 
though 1.5-T unit MRI is mainly used in Japan, 3.0-T unit MRI would be becoming standard. SSFP MRI examination with 3.0-T unit should be conducted in the future study.

In conclusion, noncontrast-enhanced SSFP MRI with a spatially selective IR pulse can improve the visibility of renal corticomedullary differentiation even in patients with advanced CKD patients. This new MRI method could detect early morphological changes in patients with CKD and would be useful for differentiating CKD etiologies in the future.

Acknowledgements We thank Kyuutaro Koyama and Hiroaki Sano for their excellent technical assistance. This work was supported by a grant from the Kidney Foundation to T. Otsuka.

\section{Compliance with ethical standards}

Conflict of interest The authors have declared that no conflict of interest exists.

Informed consent All of the participants provided written informed consent.

Open Access This article is distributed under the terms of the Creative Commons Attribution 4.0 International License (http://crea tivecommons.org/licenses/by/4.0/), which permits unrestricted use, distribution, and reproduction in any medium, provided you give appropriate credit to the original author(s) and the source, provide a link to the Creative Commons license, and indicate if changes were made.

\section{References}

1. Cheung CM, Shurrab AE, Buckley DL, Hegarty J, Middleton RJ, Mamtora H, Kalra PA. MR-derived renal morphology and renal function in patients with atherosclerotic renovascular disease. Kidney Int. 2006;69:715-22.

2. Beland MD, Walle NL, Machan JT, Cronan JJ. Renal cortical thickness measured at ultrasound: is it better than renal length as an indicator of renal function in chronic kidney disease? AJR Am J Roentgenol. 2010;195:W146-9.

3. Emamian SA, Nielsen MB, Pedersen JF. Intraobserver and interobserver variations in sonographic measurements of kidney size in adult volunteers: a comparison of linear measurements and volumetric estimates. Acta Radiol. 1995;36:399-401.

4. Semelka RC, Corrigan K, Ascher SM, Brown JJ, Colindres RE. Renal corticomedullary differentiation: observation in patients with differing serum creatinine levels. Radiology. 1994;190:149-52.

5. Lee VS, Kaur M, Bokacheva L, Chen Q, Rusinek H, Thakur R, Moses D, Nazzaro C, Kramer EL. What causes diminished corticomedullary differentiation in renal insufficiency? J Magn Reson Imaging. 2007;25:790-5.

6. Grobner T, Prischl FC. Gadolinium and nephrogenic systemic fibrosis. Kidney Int. 2007;72:260-4.

7. Kanki A, Ito K, Tamada T, Noda Y, Yamamoto A, Tanimoto D, Sato T, Higaki A. Corticomedullary differentiation of the kidney: evaluation with noncontrast-enhanced steady-state free precession (SSFP) MRI with time-spatial labeling inversion pulse (Time-SLIP). J Magn Reson Imaging. 2013;37:1178-81.

8. Noda Y, Ito K, Kanki A, Tamada T, Yamamoto A, Yasokawa K, Higaki A. Measurement of renal cortical thickness using noncontrast-enhanced steady-state free precession MRI with spatially selective inversion recovery pulse: association with renal function. J Magn Reson Imaging. 2015;41:1615-21.

9. KDIGO 2012 clinical practice guideline for the evaluation and management of chronic kidney disease. Kidney Int Suppl. 2013;3:19-62.

10. Japanese Society of Nephrology. Evidence-based clinical practice guideline for CKD 2013. Clin Exp Nephrol. 2014;18:346-423.

11. Matsuo S, Imai E, Horio M, Yasuda $Y$, Tomita K, Nitta K, Yamagata K, Tomino Y, Yokoyama H, Hishida A. Revised equations for estimated GFR from serum creatinine in Japan. Am J Kidney Dis. 2009;53:982-92.

12. DuBois D, DuBois E. A formula to estimate the approximate surface area if height and weight be known. Arch Intern Med. 1916;17:863-71.

13. American Diabetes Association. Diagnosis and classification of diabetes mellitus. Diabetes Care. 2010;33(Suppl 1):S62-9.

14. Musso CG, Oreopoulos DG. Aging and physiological changes of the kidneys including changes in glomerular filtration rate. Nephron Physiol. 2001;119(suppl 1):1-5.

15. Garasto S, Fusco S, Corica F, Rosignuolo M, Marino A, Montesanto A, De Rango F, Maggio M, Mari V, Corsonello A, Lattanzio F. Estimating glomerular filtration rate in older people. Biomed Res Int. 2014;2014:916542.

16. Mounier-Vehier C, Lions C, Devos P, Jaboureck O, Willoteaux S, Carre A, Beregi J. Cortical thickness: an early morphological marker of atherosclerotic renal disease. Kidney Int. 2002;61:591-8.

17. Kanki A, Ito K, Tamada T, Noda Y, Yamamoto A, Higaki A, Sato T, Yasokawa K, Abe T, Yoshida K. Renal corticomedullary differentiation by non-contrast-enhanced MR imaging with a spatially selective IR pulse at various inversion times: comparison with fast asymmetric spin echo (FASE) and steady-state free-precession (SSFP). Magn Reson Med Sci. 2014;13:127-31.

18. Noda Y, Kanki A, Yamamoto A, Higashi H, Tanimoto D, Sato T, Higaki A, Tamada T, Ito K. Age-related change in renal corticomedullary differentiation: evaluation with noncontrast-enhanced steady-state free precession (SSFP) MRI with spatially selective inversion pulse using variable inversion time. J Magn Reson Imaging. 2014;40:79-83.

19. Wang X, Vrtiska TJ, Avula RT, Walters LR, Chakkera HA, Kremers WK, Lerman LO, Rule AD. Age, kidney function, and risk factors associate differently with cortical and medullary volumes of the kidney. Kidney Int. 2014;85:677-85.

20. Liu Y. Cellular and molecular mechanisms of renal fibrosis. Nat Rev Nephrol. 2011;7:684-96.

21. Vallon V, Thomson SC. Renal function in diabetic disease models: the tubular system in the pathophysiology of the diabetic kidney. Annu Rev Physiol. 2012;74:351-75.

22. Thomson SC, Vallon V, Blantz RC. Kidney function in early diabetes: the tubular hypothesis of glomerular filtration. Am J Physiol Renal Physiol. 2004;286:F8-15.

23. Nguyen MT, Maynard SE, Kimmel PL. Misapplications of commonly used kidney equations: renal physiology in practice. Clin J Am Soc Nephrol. 2009;4:528-34.

24. Geddes CC, Woo YM, Brady S. Glomerular filtration rate-what is the rationale and justification of normalizing GFR for body surface area? Nephrol Dial Transplant. 2008;23:4-6. 